\title{
High-pressure infrared spectroscopy: Tuning of the low-energy excitations in correlated electron systems
}

\author{
I. Kézsmárki, ${ }^{1}$ R. Gaál, ${ }^{2}$ C. C. Homes,${ }^{3}$ B. Sípos, ${ }^{2}$ H. Berger,${ }^{2}$ S. Bordács,${ }^{1}$ G. Mihály, ${ }^{1}$ and L. Forró ${ }^{2}$ \\ ${ }^{1}$ Department of Physics, Budapest University of Technology and Economics, 1111 Budapest, Hungary \\ ${ }^{2}$ Ecole Politechnique Federale, Lausanne CH-1015, Switzerland \\ ${ }^{3}$ Condensed Matter Physics and Materials Sciences Department, Brookhaven National Laboratory, Upton New York, 11973, USA
}

(Received 20 July 2007; revised manuscript received 4 October 2007; published 21 November 2007)

\begin{abstract}
We have extended the range of the high-pressure optical spectroscopy to the far-infrared region keeping the accuracy of ambient-pressure experiments. The developed method offers a powerful tool for the study of pressure-induced phase transitions and electronic-structural changes in correlated electron systems as the optical pressure cell, equipped with large free-aperture diamond window, allows the measurement of optical reflectivity down to $\omega \approx 20-30 \mathrm{~cm}^{-1}$ for hydrostatic pressures up to $p \approx 26 \mathrm{kbar}$. The efficiency of the technique is demonstrated by the investigation of the two-dimensional charge-density-wave $1 T-\mathrm{TaS}_{2}$ whose electronic structure shows high sensitivity to external pressure. The room-temperature semimetallic phase of $1 T-\mathrm{TaS}_{2}$ is effectively extended by application of pressure and stabilized as the ground state above $p$ $=14 \mathrm{kbar}$. The corresponding fully incoherent low-energy optical conductivity is almost temperature independent below $T=300 \mathrm{~K}$. For intermediate pressures, the onset of the low-temperature insulating phase is reflected by the sudden drop of the reflectivity and by the emergence of sharp phonon resonances.
\end{abstract}

DOI: 10.1103/PhysRevB.76.205114

PACS number(s): 71.30.+h, 71.27.+a, 78.20.-e

\section{INTRODUCTION}

The application of hydrostatic pressure offers a clean and controllable way to fine tune the electronic and magnetic properties of solids via the change of their bandwidth or dimensionality or by altering the energy scale of the relevant interactions, such as magnetic exchange interactions or the electron-phonon coupling. The possibility to gain spectroscopic information about the electronic-structural changes upon pressure-induced phase transitions has recently attracted broad interest. As one of the earliest trials, the possible metallization of solid hydrogen was studied under extremely high pressures $(p \sim 1500 \mathrm{kbar})$ by infrared absorption and reflectivity techniques. ${ }^{1,2}$ In correlated electron systems, due to the strong competition between neighboring thermodynamic phases, moderate pressures can effectively induce phase transitions. Therefore, hydrostatic pressure has been successfully applied to investigate the phase diagram of correlated electron materials, especially for compounds located in the vicinity of an insulator-metal phase boundary. The pressure-driven collapse of the insulating state has been recently followed by infrared spectroscopy in the colossal magnetoresistance manganite $\mathrm{La}_{1-x} \mathrm{Ca}_{x} \mathrm{MnO}_{3},{ }^{3}$ in Mott insulators such as $\mathrm{YNiO}_{3}$ (Ref. 4) and $\mathrm{V}_{3} \mathrm{O}_{5},{ }^{5}$ in the band insulator $\mathrm{YH}_{3},{ }^{6}$ and in the chargedensity-wave rare-earth tritellurides. ${ }^{3}$ Besides the phase transition phenomena, strong pressure-induced variation of the electronic structure has been reported for $\mathrm{CeSb},{ }^{7}$ $\beta$ - $\mathrm{Na}_{0.33} \mathrm{~V}_{2} \mathrm{O}_{5},{ }^{8}$ silane, ${ }^{9}$ etc. All of these experiments were performed using diamond anvil cells (DACs) which allowed the generation of hydrostatic pressure up to $p \approx 260 \mathrm{kbar}$. The disadvantage of DACs is their pressure-limited small aperture, typically $\sim 100 \mu \mathrm{m}$ in diameter, which introduces a low-energy cutoff ( $\mathrm{a}$ few hundreds of $\mathrm{cm}^{-1}$ ) due to the diffraction effects. Consequently, these investigations were restricted to the mid-infrared and higher-energy range, al- though owing to its high transparency, diamond is an ideal optical-window material down to microwave frequencies. ${ }^{10}$

\section{EXPERIMENTAL SETUP}

In strongly correlated electron systems, where usually the low-energy excitations are of interest, spectral information about the far-infrared region is highly desirable. Focusing on materials which show high sensitivity to external pressure, we have developed a conceptually different type of optical pressure cell which extends the low-energy limit of the infrared experiments by more than one decade as it is applicable typically down to $\omega \approx 20-30 \mathrm{~cm}^{-1}$. In our design, this improvement is the result of the large free aperture of the window $(d=1.5 \mathrm{~mm})$ which restricts the maximum applied pressure $p \approx 26 \mathrm{kbar}$ at the same time. On the basis of reflectivity measurements performed on the two-dimensional charge-density-wave material $1 T-\mathrm{TaS}_{2}$, we demonstrate that this type of pressure cell allows the determination of the absolute value of the reflectivity even in the far-infrared region. Furthermore, its precision is comparable to that of standard ambient-condition reflectivity experiments. Our design for the diamond-window based self-clamping-type pressure cell is shown in Fig. 1(a). The light access is achieved through a type IIA diamond window fixed at the lateral part of the metallic body with the beam being perpendicular to the horizontal axis of the cell. In order to determine the absolute value of reflectivity for the diamond-sample interface $R_{d s}$, the window should have wedged plane surfaces which facilitate the measurement of reference signal. It is also sufficient to eliminate interference fringes coming from multiple reflections. Besides the optical access, an electrical lead through is also implemented, which facilitates either the in situ monitoring of pressure by a resistive sensor or the simultaneous measurement of the sample resistivity during the pressure runs. We use kerosene as a pressure medium and an 


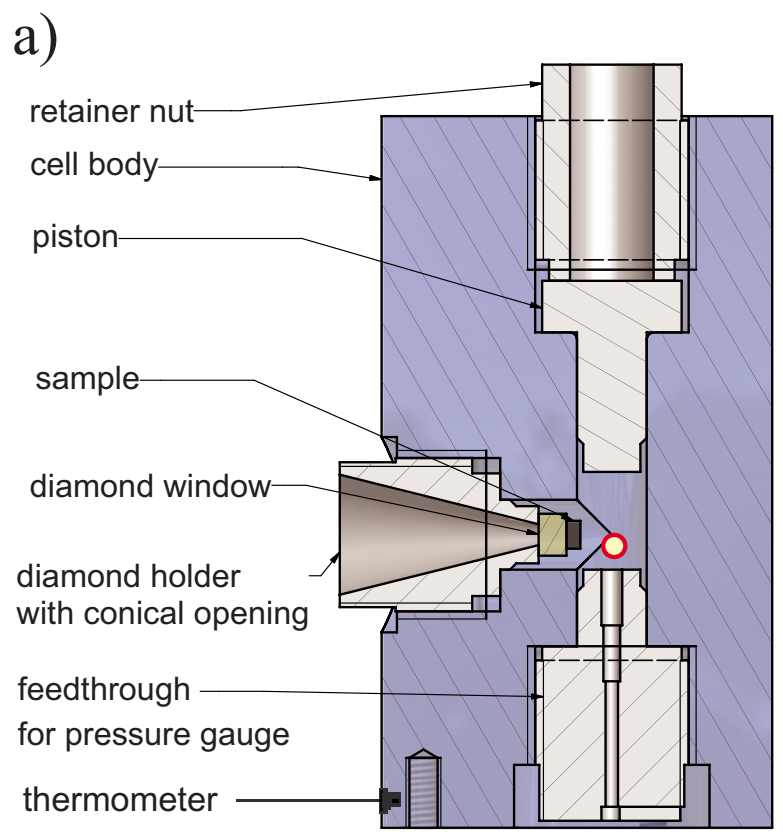

b)

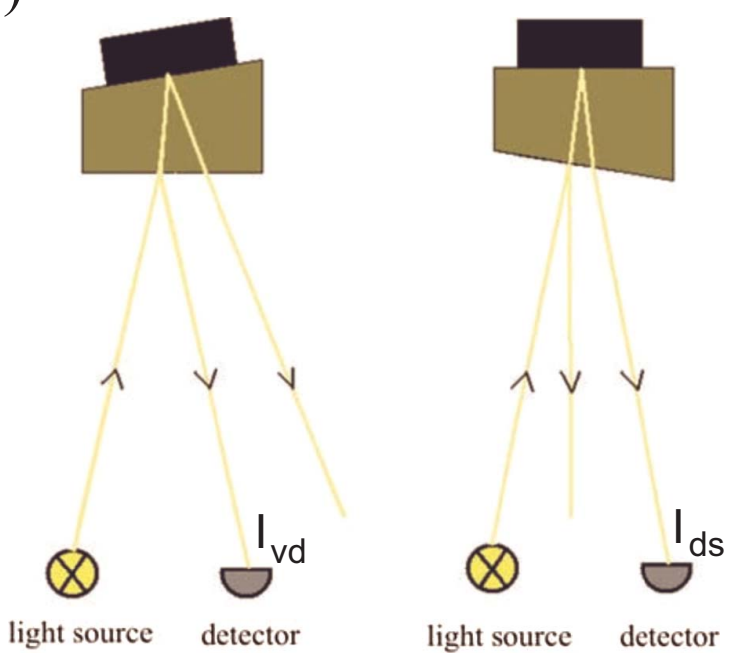

FIG. 1. (Color online) Our home-designed optical pressure cell: steel body equipped with a type IIA diamond window. The upper panel shows the prorata drawing of the cell. The sample is mounted on the inner surface of the diamond window which has $d$ $=1.5 \mathrm{~mm}$ free aperture. The window holder is removable and has a conical opening of $\sim 28^{\circ}$. The location of the pressure sensor indicated by an open circle close to the sample is connected via a lead trough. The position of the thermometer at the bottom of the cell is also shown. As demonstrated in the lower panel, the angle of wedging between the two surfaces of the window allows for a clean separation of the reflections from the vacuum-diamond and diamond-sample interfaces $\left(I_{v d}\right.$ and $I_{d s}$, respectively) and thus facilitates reference measurements.

InSb sensor as pressure gauge located about $2 \mathrm{~mm}$ far from the sample on the top of the lead trough. The sealing between the diamond window and its holder is a thin indium foil which is removed from the free surface of the window after applying a tiny pressure, typically $0.5 \mathrm{kbar}$. For low- temperature experiments, the pressure cell is attached to the cold finger of a $\mathrm{He}$ flow cryostat, and the thermalization between the sample and the thermometer installed at the bottom of the cell is ensured by a radiation shield.

As followed in the sketch of Fig. 1(b), the intensity reflected back from the vacuum-diamond and diamond-sample interfaces $\left(I_{v d}\right.$ and $I_{d s}$, respectively) can be detected separately by a few degrees (typically $\sim 7^{\circ}$ ) of rotation of the cell around its vertical axis due to the wedging of the diamond. (A wedging angle of $2^{\circ}$ results in $\sim 10^{\circ}$ angular deviation between the two reflected beams, which together with the finite beam size and the rotation of the cell, requires an opening angle of $\sim 28^{\circ}$ for the conical bore on the diamond holder in our optical arrangement.) The nearly normal incidence is still held for the both positions. Correspondingly, the reflectivity of the sample relative to the diamond can be obtained from the measured intensities, ${ }^{11}$

$$
R_{d s}(\omega)=\frac{R_{v d}(\omega)}{\left[1-R_{v d}(\omega)\right]^{2}} \frac{I_{d s}(\omega)}{I_{v d}(\omega)},
$$

where $R_{v d}\left(\equiv R_{d}\right)$ is the absolute reflectivity of the diamond. The $R_{v d}(\omega) /\left[1-R_{v d}(\omega)\right]^{2}$ prefactor can be calculated from the well-documented refractive index of diamond $\hat{n}_{d} \cdot{ }^{10,12-15}$ High-quality, such as type IIA, optical diamonds show no significant absorption up to $\omega \approx 40000 \mathrm{~cm}^{-1}$. On the other hand, the effect of weak absorption introduces only another frequency-dependent factor into Eq. (1) (which can be directly determined from the transmittance of the window), but its influence on $R_{v d}$ and $R_{d s}$ can be usually neglected. If $R_{d s}(\omega)$ is measured over a sufficiently broad range of energy, the complex refractive index of the sample relative to the diamond $\hat{n}_{d s}(\omega)$ can be obtained by the Kramers-Kronig analysis. Since $\hat{n}_{d}(\omega)$ is purely real and shows a monotonous increase of about $10 \%$ up to $\omega \approx 40000 \mathrm{~cm}^{-1}$, it is straightforward to calculate the complex dielectric response of the sample relative to the vacuum. This analysis fails only for the multiphonon absorption bands of the diamond located in the range of $\omega=1500-2700 \mathrm{~cm}^{-1} .{ }^{15}$ We note at this point that, additional to the absorption of the diamond above $\omega$ $\approx 40000 \mathrm{~cm}^{-1}$, the high-energy limit of this method is determined by the roughness and/or planarity of the diamondsample interface $\delta_{d s}$. Therefore, special care should be taken for the proper matching between the window and the sample in order to eliminate interference and diffraction effects inherently appearing for wavelength shorter than $\delta_{d s}$. To prevent the development of a kerosene film between the sample and the diamond, the diamond-sample duet was covered by indium foil before assembling the cell.

\section{RESULTS AND DISCUSSION}

In the following, we shall present the high-pressure optical study of the two-dimensional charge-density-wave (CDW) system $1 T-\mathrm{TaS}_{2}$ carried out by the above described pressure cell. At ambient pressure and room temperature, $1 T-\mathrm{TaS}_{2}$ is a bad metal or semimetal characterized by a high and almost temperature independent resistivity $(\rho$ $\approx 10^{-3} \Omega \mathrm{cm}$ ) and by a fully incoherent low-energy optical 


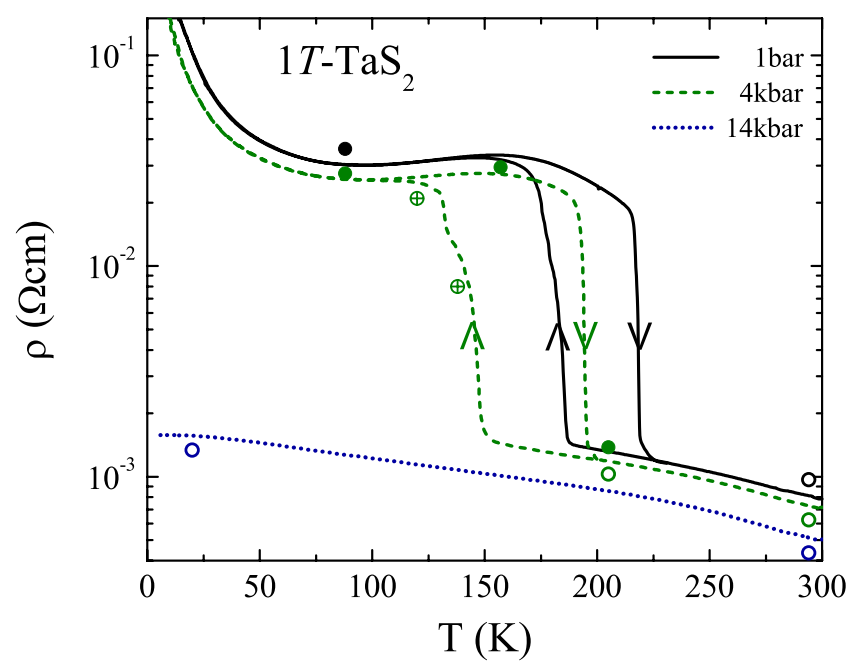

FIG. 2. (Color online) Temperature dependence of the resistivity for three selected pressures in $1 T-\mathrm{TaS}_{2}$. At each pressure, infrared reflectivity was measured at temperatures labeled by different symbols: $\bigcirc, \boldsymbol{\ominus}$, and $\oplus$ correspond to points in the pressure-temperature phase diagram, which are characterized by the reflectivity spectrum as the room-temperature phase, low-temperature phase, or as a mixed state, respectively. At $p=1 \mathrm{bar} / 14 \mathrm{kbar}$, the corresponding symbols are shown above or below the resistivity curve. For $p$ $=4 \mathrm{kbar}$, the reflectivity measurements performed in cooling run are indicated below, while those in warming up are shown above the $\rho(T)$ curve.

conductivity. ${ }^{17}$ With lowering the temperature, the material goes through a first-order phase transition at $T_{\text {lock }}=180 \mathrm{~K}$ which is clearly manifested in the electronic transport as it is

a)

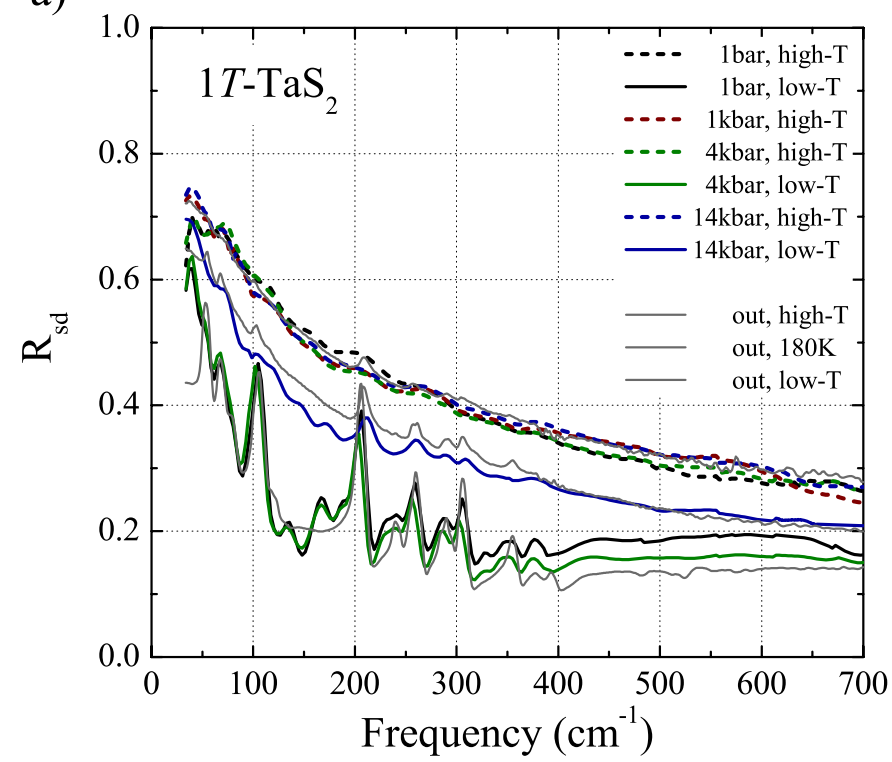

accompanied by a jumplike enhancement of the resistivity ${ }^{16}$ and by the opening of a soft gap $\Delta \approx 800 \mathrm{~cm}^{-1}$ in the optical conductivity. ${ }^{17}$ Recent photoemission experiments also reflect that the electronic-structural changes are dominantly restricted to the low-energy region. ${ }^{18-21}$ On the other hand, due to its two-dimensional CDW ordering and the large electronphonon coupling, ${ }^{20,21}$ the material shows extremely high sensitivity to external pressure. ${ }^{16}$ Therefore, $1 T-\mathrm{TaS}_{2}$ offers an ideal playground for the study of the pressure-induced electronic and structural changes by infrared spectroscopy.

Prior to the optical experiments under pressure, we have investigated the pressure-temperature phase diagram of the material by means of dc resistivity. The results are shown in Fig. 2 for three selected pressures $p=1$ bar, 4 kbar, and 14 kbar. In agreement with early data, ${ }^{16}$ the low-temperature insulating phase is strongly suppressed although the resistivity is almost unchanged in either of the phases. Already at $p=4 \mathrm{kbar}$, the transition is shifted to $T_{\text {lock }} \approx 150 \mathrm{~K}$ and the hysteresis is significantly broadened, ${ }^{22}$ while at $p=14 \mathrm{kbar}$, the high-temperature phase is extended to the lowest temperatures. This semimetallic state exhibits nearly temperature independent charge transport as the resistivity increases only by a factor of $\sim 3$ below $T=300 \mathrm{~K}$. We note here that the resistivity in the insulating phase also shows anomalous behavior which may be attributed to the Mott-Anderson localization effects together with the opening of the pseudogap $\Delta \approx 800 \mathrm{~cm}^{-1}$.

The far-infrared reflectivity of $1 T-\mathrm{TaS}_{2}$ was investigated below room temperature for various pressures $p=1$ bar, $1 \mathrm{kbar}, 4 \mathrm{kbar}$, and $14 \mathrm{kbar}$ in the photon energy range of $\omega=30-700 \mathrm{~cm}^{-1}$, as shown in Fig. 3. For verification of the results, besides the $R_{d s}(\omega)$ spectra measured inside of the pressure cell, Fig. 3(a) also displays spectra evaluated from

b)

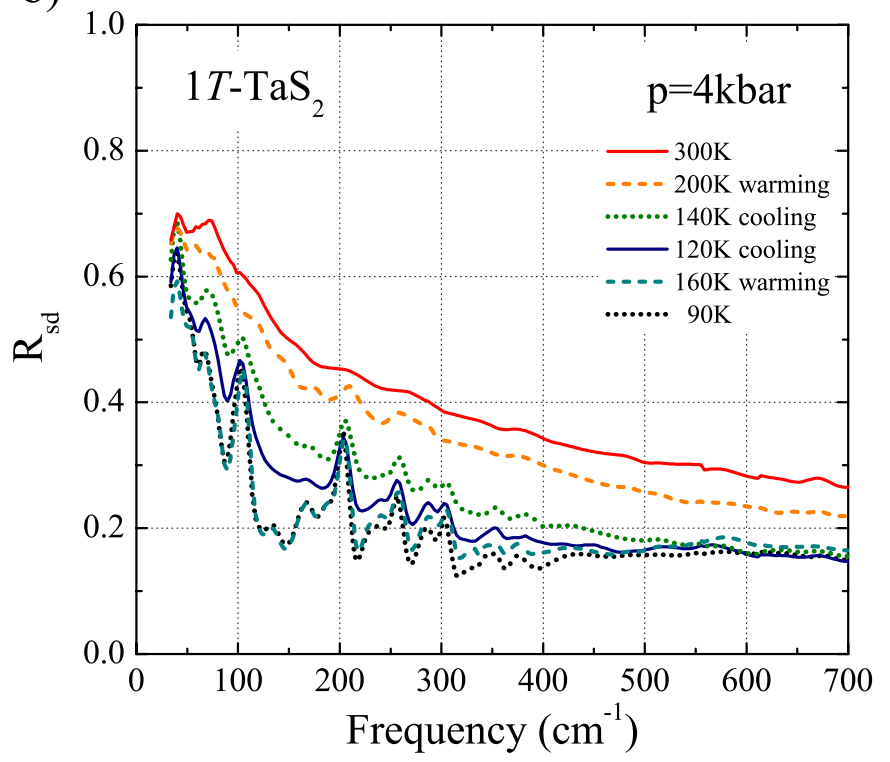

FIG. 3. (Color online) Far-infrared reflectivity of $1 T-\mathrm{TaS}_{2}$ relative to diamond $R_{d s}(\omega)$ over a broad range of hydrostatic pressure and temperature. Left panel: reflectivity spectra measured in the room-temperature and the low-temperature phases of $1 T-\mathrm{TaS}_{2}$ at various pressures. Temperatures corresponding to the low-temperature measurements can be followed in Fig. 2. For the verification of the results obtained within the pressure cell, $R_{d s}(\omega)$ spectra calculated from the ambient-pressure absolute reflectivity (reproduced from Ref. 17) are also plotted. Right panel: far-infrared reflectivity of $1 T-\mathrm{TaS}_{2}$ below room temperature at $p=4 \mathrm{kbar}$. 
the ambient-pressure absolute reflectivity $R_{s}(\omega)$ reproduced from Ref. 17. (For these data, the complex refractive index $\hat{n}_{s}$ was obtained by the Kramers-Kronig transformation and $R_{d s}$ was calculated with use of the Fresnel equation from $\hat{n}_{s}$ and $\hat{n}_{d}$.) The ambient-pressure data measured inside and out of the pressure cell show fairly good agreement both at room and low temperature verifying the applicability of our method for the fully quantitative measurement of reflectivity under pressure even in the hardly accessible far-infrared spectral range.

Similarly to the resistivity, the room-temperature reflectivity of the sample does not show significant variation up to $p=14 \mathrm{kbar}$. The reflectivity spectrum in the low-temperature insulating phase is reduced and exhibits sharp phonon resonances for $\omega<400 \mathrm{~cm}^{-1}$. Its variation with pressure is also negligible for $p=1$ bar -4 kbar and probably the same remains valid, as long as the insulating phase is not completely suppressed. On the other hand, at $p=14 \mathrm{kbar}$, when the semimetallic phase becomes the ground state, the lowtemperature reflectivity is reminiscent to that one at room temperature. Although it is somewhat reduced in absolute value, the spectral shape is still semimetalliclike and the phonon modes remain almost completely screened. For comparison, we also plotted the reflectivity measured out of the cell just above $T_{\text {lock }}=180 \mathrm{~K}$. As expected from the tiny variation of the resistivity at $p=14 \mathrm{kbar}$ below room temperature, the two spectra are close to each other over the whole farinfrared range.

The temperature dependence of $R_{d s}(\omega)$ has been studied in more details, especially in the hysteretic region of the transition, for the intermediate pressure $p=4 \mathrm{kbar}$ [see Fig. $3(\mathrm{~b})$ ]. The spectra follow the tendency of the $R(T)$ curve both in the cooling and warming runs, as depicted by symbols plotted simultaneously with the resistivity curves. Except for $T=140$ and $120 \mathrm{~K}$ in cooling down, where the coexistence of the two phases was observed in the reflectivity, $R_{d s}(\omega)$ spectra purely showed either the semimetallic or the insulating character.

The nature and the microscopic order parameter of the phase transition at $T_{\text {lock }}$ is still under debate. Although it has a strong first-order character (for each pressure $p<14 \mathrm{kbar}$ ) in sense of broad hysteresis in the temperature loops, it is associated with a rather small, about 1 order of magnitude, jump in the resistivity. Furthermore, the tendency of the temperature dependence on the two sides of $T_{\text {lock }}(p)$ does not show significant change. The essence of the change in the electronic states as captured by the optical spectra is the radi- cal spectral-weight transfer from $\omega \leq 5000 \mathrm{~cm}^{-1}$ to the higher-energy region. This indicates that the charge carriers exhibiting incoherent, hoppinglike transport in the semimetallic phase are further localized. However, we think that the transition is primarily driven by the lattice degrees of freedom for two reasons: (i) the electronic character of the transition, followed by the resistivity curves, is not altered by the pressure $p<14 \mathrm{kbar}$, only $T_{\text {lock }}(p)$ is significantly reduced; (ii) the low-temperature spectra are also nearly pressure independent as far as the insulating ground state exists. Thus, it is very likely that the charge gap characterizing the change in the electronic system is also unchanged by pressure in strong contrast to $T_{\text {lock }}(p)$.

\section{SUMMARY}

In conclusion, we have extended the range of the highpressure optical spectroscopy to the far-infrared region keeping the accuracy of ambient-pressure experiments. For this purpose, we have developed an optical pressure cell equipped with a wedged diamond window of large free aperture by which reflectivity spectra can be measured down to $\omega \approx 20-30 \mathrm{~cm}^{-1}$ for hydrostatic pressures up to $p=26 \mathrm{kbar}$. This technique, which is strongly based on the excellent optical properties of high-quality (type IIA) diamonds, is well suited for the study of the systematics of bandwidthcontrolled insulator-metal transitions in interacting electron systems via the pressure-induced changes in their low-energy electronic structure. Its applicability was demonstrated by the optical study of the two-dimensional charge-densitywave $1 T-\mathrm{TaS}_{2}$. We have pointed out that the roomtemperature semimetallic phase can be effectively extended by external pressure and stabilized as the ground state above $p=14$ kbar. Furthermore, the corresponding fully incoherent low-energy optical conductivity is almost temperature independent below $T=300 \mathrm{~K}$. For intermediate pressures, the onset of the low-temperature insulating phase is reflected by the sudden drop of the reflectivity and by the emergence of sharp phonon resonances.

\section{ACKNOWLEDGMENTS}

The authors are grateful to L. Mihály for useful comments and discussions. They are also greatly indebted to L. V. Gasparov for sending recently published ambient-pressure reflectivity spectra. This work was supported by the Hungarian Scientific Research Funds OTKA under Grant Nos. F61413 and K62441, and Bolyai 00239/04, by the Swiss NSF, and its NCCR "MaNEP," and by the DOE under Contract No. DEAC02-98CH10886.
${ }^{1}$ H. K. Mao, R. J. Hemley, and M. Hanfland, Phys. Rev. Lett. 65, 484 (1990).

${ }^{2}$ R. J. Hemley, H. K. Mao, A. F. Goncharov, M. Hanfland, and V. V. Struzhkin, Phys. Rev. Lett. 76, 1667 (1996).

${ }^{3}$ A. Sacchetti, M. C. Guidi, E. Arcangeletti, A. Nucara, P. Calvani, M. Piccinini, A. Marcelli, and P. Postorino, Phys. Rev. Lett. 96, 035503 (2006).
${ }^{4}$ J. L. Garcia-Munoz, M. Amboage, M. Hanfland, J. A. Alonso, M. J. Martinez-Lope, and R. Mortimer, Phys. Rev. B 69, 094106 (2004).

${ }^{5}$ L. Baldassarre, A. Perucchi, E. Arcangeletti, D. Nicoletti, D. Di Castro, P. Postorino, V. A. Sidorov, and S. Lupi, Phys. Rev. B 75, 245108 (2007).

${ }^{6}$ A. Ohmura, A. Machida, T. Watanuki, K. Aoki, S. Nakano, and 
K. Takemura, Phys. Rev. B 73, 104105 (2006).

${ }^{7}$ T. Nishi, S. Kimura, T. Takahashi, Y. Mori, Y. S. Kwon, H. J. Im, and H. Kitazawa, Phys. Rev. B 71, 220401(R) (2005).

${ }^{8}$ C. A. Kuntscher, S. Frank, I. Loa, K. Syassen, T. Yamauchi, and Y. Ueda, Phys. Rev. B 71, 220502(R) (2005).

${ }^{9}$ L. Sun, A. L. Ruoff, C. S. Zha, and G. Stupian, J. Phys. D 18, 8573 (2006).

${ }^{10}$ D. F. Edwards and H. R. Philipp, in Handbook of Optical Constants of Solids, edited by E. D. Palik (Academic, Orlando, 1985), pp. 665-673.

${ }^{11}$ The validity of the equation is restricted to the case when the vacuum-diamond and diamond-sample interfaces can be treated independently (as their distance is much larger than the wavelength of the light), and multiple reflections between the two sides of the window can be excluded. Both conditions are fulfilled in the present arrangement.

${ }^{12}$ H. R. Philipp and E. A. Taft, Phys. Rev. 136, A1445 (1964).

${ }^{13}$ D. F. Edwards and E. Ochoa, J. Opt. Soc. Am. 71, 607 (1981).

${ }^{14}$ A. B. Djurisic and E. H. Li, Appl. Opt. 37, 7273 (1998).

${ }^{15}$ M. E. Thomas, W. J. Tropf, and A. Szpak, Diamond Films Tech- nol. 5, 159 (1995), and references therein.

${ }^{16}$ T. Tani, T. Osada, and S. Tanaka, Solid State Commun. 22, 269 (1977).

${ }^{17}$ L. V. Gasparov, K. G. Brown, A. C. Wint, D. B. Tanner, H. Berger, G. Margaritondo, R. Gaal, and L. Forro, Phys. Rev. B 66, 094301 (2002).

${ }^{18}$ F. Zwick, H. Berger, I. Vobornik, G. Margaritondo, L. Forro, C. Beeli, M. Onellion, G. Panaccione, A. Taleb-Ibrahimi, and M. Griono, Phys. Rev. Lett. 81, 1058 (1998).

${ }^{19}$ L. Perfetti, T. A. Gloor, F. Mila, H. Berger, and M. Grioni, Phys. Rev. B 71, 153101 (2005).

${ }^{20}$ L. Perfetti, P. A. Loukakos, M. Lisowski, U. Bovensiepen, H. Berger, S. Biermann, P. S. Cornaglia, A. Georges, and M. Wolf, Phys. Rev. Lett. 97, 067402 (2006).

${ }^{21}$ F. Clerc, C. Battaglia, M. Bovet, L. Despont, C. Monney, H. Cercellier, M. G. Garnier, P. Aebi, H. Berger, and L. Forro, Phys. Rev. B 74, 155114 (2006).

${ }^{22}$ In our convention, $T_{\text {lock }}$ is defined by the temperature, the onset of the transition in cooling down. 